\title{
ORIGINAL
}

\section{Effect of interplay between age and low-flow duration on neurologic outcomes of extracorporeal cardiopulmonary resuscitation}

\author{
Hsi-Yu Yu, Chih-Hsien Wang, Nai-Hsin Chi, Shu-Chien Huang, Heng-Wen Chou, Nai-Kuan Chou \\ and Yih-Sharng Chen* ${ }^{*}$
}

(c) 2018 The Author(s)

\begin{abstract}
Purpose: Caseloads of extracorporeal cardiopulmonary resuscitation (ECPR) have increased considerably, and hospital mortality rates remain high and unpredictable. The present study evaluated the effects of the interplay between age and prolonged low-flow duration (LFD) on hospital survival rates in elderly patients to identify subgroups that can benefit from ECPR.

Methods: Adult patients who received ECPR in our institution (2006-2016) were classified into groups 1, 2, and 3 $(18-65,65-75$, and $>75$ years, respectively). Data regarding ECPR and adverse events during hospitalization were collected prospectively. The primary end point was favorable neurologic outcome (cerebral performance category 1 or 2) at hospital discharge.

Results: In total, 482 patients were divided into groups 1,2 , and $3(70.5 \%, 19.3 \%$, and 10.2\%, respectively). LFDs were comparable among the groups $(40.3,41.0$, and 44.3 min in groups 1,2 , and $3, P=0.781,0.231$, and 0.382 , respectively). Favorable neurologic outcome rates were nonsignificantly lower in group 3 than in the other groups $(27.6 \%, 24.7 \%$, and $18.4 \%$ for group 1, 2, and 3, respectively). Subgroup analysis revealed that the favorable neurologic outcome rates in group 1 were $36.7 \%, 25.4 \%$, and $13.0 \%$ for LFDs of $<30,30-60$, and $>60$ min, respectively $(P=0.005)$; in group 2 , they were $32.1 \%, 21.2 \%$, and $23.1 \%$, respectively $(P=0.548)$; in group 3 they were $25.0 \%, 20.8 \%$, and $0.0 \%$, respectively $(P=0.274)$.

Conclusion: On emergency consultation for ECPR, age and low-flow duration should be considered together to predict neurologic outcome.

Keywords: Extracorporeal membrane oxygenation, Cardiopulmonary resuscitation, Extracorporeal cardiopulmonary resuscitation, Low-flow duration, Age
\end{abstract}

\footnotetext{
*Correspondence: yschen1234@gmail.com
}

Department of Surgery, National Taiwan University Hospital, and College

of Medicine, National Taiwan University, No. 7, Chung-Shan South Road,

Taipei 100, Taiwan

\section{至




\section{Introduction}

Extracorporeal cardiopulmonary resuscitation (ECPR) is labor-intensive work, and the caseload has increased considerably in the past decade, accounting for one-quarter of adult extracorporeal membrane oxygenation (ECMO) cases of cardiac support [1]. ECPR is an expensive and labor-intensive mode of circulatory support, but the overall hospital survival rate for adult ECPR cases is relatively low at 29\% [1]. Thus, more than two-thirds of ECPR efforts are futile in terms of hospital survival. From the viewpoint of balance of beneficence and nonmaleficence versus autonomy, dignity for elderly patients undergoing cardiopulmonary resuscitation (CPR) [2], and limited resources and overloaded manpower in emergency ECMO services, predicting the outcome of ECMO during CPR by considering prominent risk factors is practical and mandatory. A do-not-resuscitate (DNR) order can be obtained for patients who are unlikely to benefit from ECMO support.

Age and low-flow duration (LFD) are two of the most prominent factors affecting ECPR. Several studies have shown that prolonged LFDs are significantly associated with poor hospital outcomes for ECPR [3, 4], with an approximate survival rate of only $10-17 \%$ if LFDs exceed $60 \mathrm{~min}$. Animal experiments have indicated that old and young animals present different degrees of neuroinflammation and apoptosis reaction after brain ischemic damage $[5,6]$. The number of elderly patients being referred for ECMO support when undergoing CPR has increased in recent years [7-10]. Although "age" has not been indicated to have a negative linear correlation with hospital survival in patients with ECPR $[7,11]$, the Good Outcome Following Attempted Resuscitation score [12] for CPR and the survival after veno-arterial-ECMO (SAVE) score [13] for ECMO both indicate that old age is a risk factor for poor hospital outcomes. The present study evaluated whether advanced old age ( $>75$ years) is a contraindication for ECPR regarding hospital outcome, with a particular focus on the effect of interplay between old age and prolonged LFDs. The results can enable the identification of subgroups among elderly patients that can benefit from ECPR. Our study results may provide objective data for deciding between ECMO and DNR and for ensuring efficient utilization of medical resources.

\section{Methods}

Our hospital is a tertiary referral hospital located in an urban area with 20 years of experience in ECMO $[14,15]$ and 12 years of experience in ECPR $[3,16,17]$. An ECPR task force was established in our institute in $2003[3,18]$. The institutional review board of our hospital approved this study (NTUH-9561707007). All data were prospectively collected and systemically reviewed, and several results have been reported in previous studies [17, 19, 20]. In the present study, we analyzed the data of all adult patients (age $\geq 18$ years) who had received ECPR at our institution between 2006 and 2016 by using a database. The patients whose index ECPR was not performed in our hospital were excluded because of unstandardized definitions of the arrest time, CPR duration, and ECMO management protocol. The patients who experienced shock necessitating ECMO in an elective or emergency situation without cardiac massage or multiple boluses of epinephrine injection were also excluded, as were those with incomplete CPR data, follow-up data, and cerebral performance category (CPC) data after hospital discharge. The patients who experienced shock necessitating CPR for $<10$ min were excluded because they did not meet the criterion of "prolonged" CPR. The contraindications of ECPR were adapted from the previously reported contraindications $[3,20]$ and are listed in Supplementary Table 1 . The equipment and management of ECMO have been described in previous studies $[3,18,20]$. The principal components of the ECMO circuit used on our patients were centrifugal pumps and an oxygenator (Medtronic, Anaheim, CA, USA; Medos, Stolberg, Germany; Maquet, Rastatt, Germany). The circuit was preorganized without priming and was then primed using saline containing $2 \mathrm{U} / \mathrm{ml}$ heparin when an ECPR call was received.

Out-of-hospital cardiac arrest (OHCA) and in-hospital cardiac arrest (IHCA) were defined as any cardiac arrest event outside and inside the hospital, respectively. For the patients who experienced OHCA, CPR and attempted defibrillation were initiated by well-trained emergency medical technicians (EMTs) in compliance with the guidelines of the American Heart Association [21]. CPR was continuously performed on the patients while they were being transported to our institution. Physicians in the emergency room consulted the ECMO team to decide whether the patient was eligible for ECPR (10 min) after advanced cardiac life support (ACLS) management. The patients who experienced IHCA were provided with onsite ACLS, or advanced life support was provided immediately by the neighboring medical personnel. An on-call CPR team responded rapidly after receiving an emergency call. After $10 \mathrm{~min}$ of initial ACLS management, which included several instances of cardiac defibrillation and bolus injection of epinephrine, the medical personnel in charge consulted the ECMO team to decide whether the patient was eligible for ECPR. LFD was defined as the interval between initiation of CPR and initiation of ECMO support.

The patients' demographic and health data before index CPR were collected immediately after ECMO setup. The Charlson Comorbidity Index (CCI) score before the 
index CPR was calculated using a formula containing 17 variables [22, 23]. Data regarding major outcomes and adverse events during hospitalization were collected during hospitalization. The major outcome was a favorable neurologic outcome (CPC 1 or 2 ) at hospital discharge. The follow-up data of the patients who survived ECPR until hospital discharge were collected through chart review and telephone interviews.

\section{Statistical analysis}

The patients were grouped according to their ages at index ECPR into three groups, namely group 1 (1865 years), group 2 (65-75 years), and group 3 (> 75 years). Continuous variables that exhibited a normal distribution were presented as the mean \pm standard deviation and were examined using Student's $t$ test. Continuous variables that exhibited a skewed distribution were presented as the median and $25-75 \%$ interquartile range and were examined using the Mann-Whitney $U$ test or Wilcoxon test. Categorical variables were analyzed using the chisquare test. The variables affecting the major outcome (i.e., hospital survival) that were significant $(P<0.15)$ in univariate analysis were included in multivariate logistic regression with backward stepwise analysis. Midterm survival was modeled using the Kaplan-Meier method, and the log-rank test was applied to test statistical significance. Statistical significance was set at $P<0.05$. All statistical analyses were performed using MedCalc statistical software version 17.8.6 (MedCalc Software, Ostend, Belgium).

\section{Results}

In total, 5664 patients experienced cardiac arrest and received CPR in our institution between 2006 and 2016 (IHCA and OHCA, 3376 and 2288 patients, respectively). Among them, 502 patients received ECMO support (ECPR cases); 20 of these patients were excluded from the present study because of incomplete data, and the remaining 482 were included (Fig. 1). The patients in groups 1, 2, and 3 accounted for $70.5 \%, 19.3 \%$, and $10.2 \%$ of all the study patients, respectively. Although the number of cases of ECPR increased in consecutive years in the study period, the proportions of the three age groups did not differ significantly between years (Supplement Fig. 1). The demographic data of the three age groups are summarized in Table 1. In brief, group 1 exhibited a significantly lower incidence of diabetes, chronic kidney disease stages 4 and 5, chronic obstructive pulmonary disease, peripheral vascular disease, and age-related stroke than those in groups 2 and 3. The mean CCI score was also lower in group 1 than in groups 2 or $3(1.34 \pm 1.77,2.14 \pm 2.00$, and $2.37 \pm 1.95$ in groups 1,2 , and 3 , respectively). All the

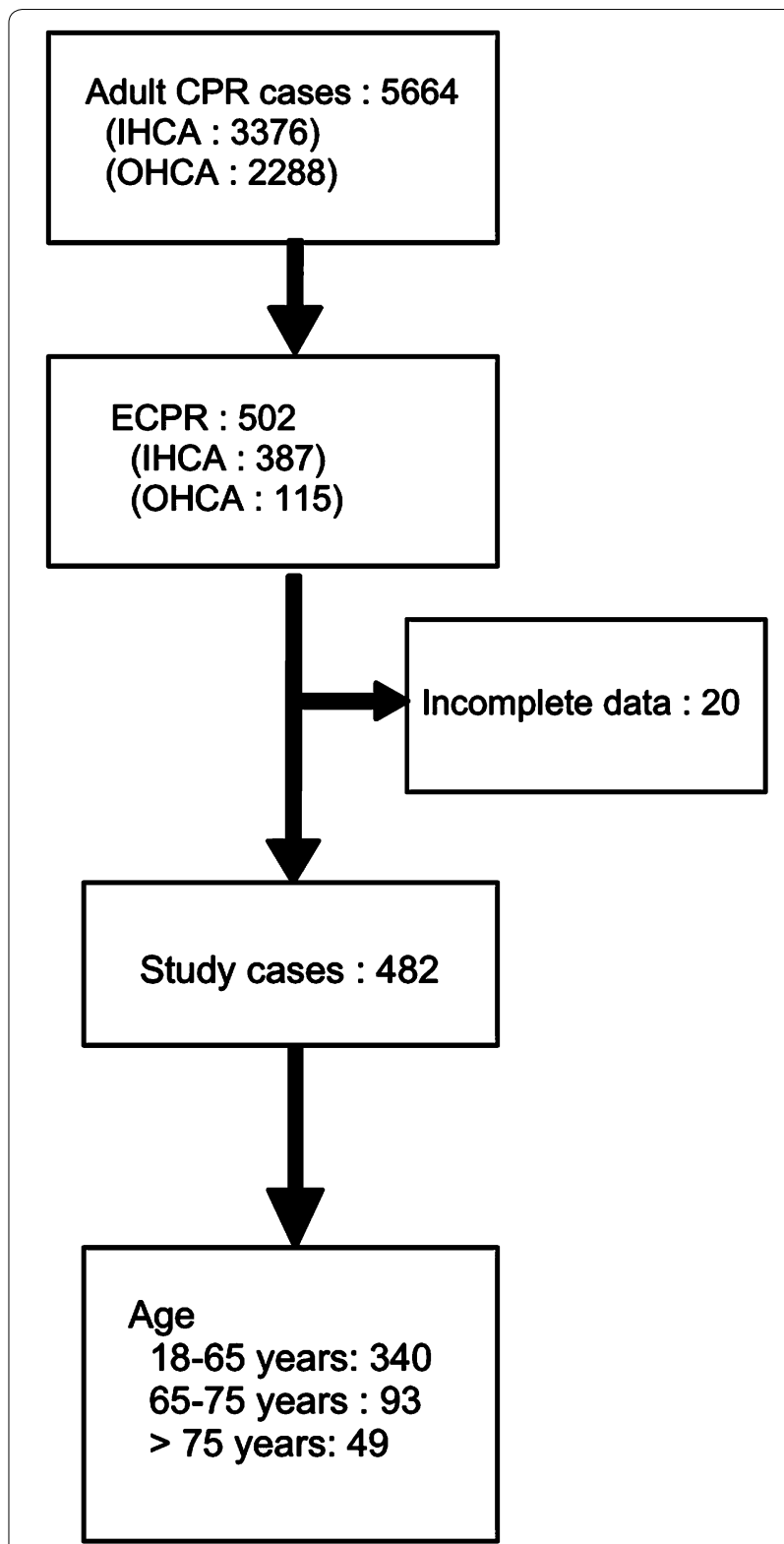

Fig. 1 Flowchart of study patients

aforementioned data revealed that the general condition before index CPR events was better in the younger patients than in the older patients.

When the underlying causes of CPR were considered, group 1 exhibited a lower percentage of patients with acute coronary syndrome (ACS) than groups 2 and 3 $(34.7 \%, 50.5 \%$, and $51.0 \%$ in groups 1,2 , and 3 , respectively; $P=0.005$ between groups 1 and 2 and $P=0.027$ between groups 1 and 3). However, group 3 exhibited a lower percentage of patients with chronic heart failure than did groups 1 and $2(18.5 \%, 14.0 \%$, and $4.1 \%$ in groups 1,2 , and 3 , respectively) (Table 1 ). The findings 
Table 1 Demographic data of patients in different age groups who received extracorporeal cardiopulmonary resuscitation (ECPR)

\begin{tabular}{|c|c|c|c|c|}
\hline & $\begin{array}{l}\text { Group 1: } 18-65 \text { years old } \\
\text { (340) }\end{array}$ & $\begin{array}{l}\text { Group 2: 65-75 years old } \\
\text { (93) }\end{array}$ & $\begin{array}{l}\text { Group 3: > } 75 \text { years old } \\
\text { (49) }\end{array}$ & $P$ \\
\hline Female gender & $71(20.9 \%)$ & $27(29.0 \%)$ & $16(32.7 \%)$ & 0.077 \\
\hline Age (years) & $49.4 \pm 11.8$ & $69.5 \pm 3.0$ & $80.2 \pm 3.9$ & $\begin{array}{l}1-2:<0.001 \\
1-3:<0.001 \\
2-3:<0.001\end{array}$ \\
\hline Diabetes mellitus & $106(31.2 \%)$ & $44(47.3 \%)$ & $26(53.1 \%)$ & $\begin{array}{l}1-2: 0.004 \\
1-3: 0.003 \\
2-3: 0.516\end{array}$ \\
\hline CKD-4, 5 & $39(11.5 \%)$ & 19 (20.4\%) & $13(26.5 \%)$ & $\begin{array}{l}1-2: 0.025 \\
1-3: 0.004 \\
2-3: 0.410\end{array}$ \\
\hline NYHA-3, 4 & $77(22.6 \%)$ & $25(26.9 \%)$ & $15(30.6 \%)$ & $\begin{array}{l}1-2: 0.394 \\
1-3: 0.221 \\
2-3: 0.640\end{array}$ \\
\hline COPD & $3(0.9 \%)$ & $2(2.2 \%)$ & $5(10.2 \%)$ & $\begin{array}{l}1-2: 0.311 \\
1-3:<0.001 \\
2-3: 0.036\end{array}$ \\
\hline Peripheral vascular disease & $12(3.5 \%)$ & $9(9.7 \%)$ & $6(12.2 \%)$ & $\begin{array}{l}1-2: 0.015 \\
1-3: 0.007 \\
2-3: 0.637\end{array}$ \\
\hline Old CVA or TIA & $19(5.6 \%)$ & $10(10.8 \%)$ & $8(16.3 \%)$ & $\begin{array}{l}1-2: 0.078 \\
1-3: 0.006 \\
2-3: 0.344\end{array}$ \\
\hline Malignancy & $15(4.4 \%)$ & $8(8.6 \%)$ & $3(6.1 \%)$ & $\begin{array}{l}1-2: 0.111 \\
1-3: 0.595 \\
2-3: 0.601\end{array}$ \\
\hline $\mathrm{CCl}$ & $1.34 \pm 1.77$ & $2.14 \pm 2.00$ & $2.37 \pm 1.95$ & $\begin{array}{l}1-2:<0.001 \\
1-3:<0.001 \\
2-3: 0.517\end{array}$ \\
\hline \multicolumn{5}{|l|}{ Main CPR causes } \\
\hline Acute coronary syndrome & $118(34.7 \%)$ & $47(50.5 \%)$ & $25(51.0 \%)$ & $\begin{array}{l}1-2: 0.005 \\
1-3: 0.027 \\
2-3: 0.975\end{array}$ \\
\hline Chronic heart failure & $63(18.5 \%)$ & $13(14.0 \%)$ & $2(4.1 \%)$ & $\begin{array}{l}1-2: 0.307 \\
1-3: 0.011 \\
2-3: 0.069\end{array}$ \\
\hline Septic shock & $29(8.5 \%)$ & $4(4.3 \%)$ & $4(8.2 \%)$ & $\begin{array}{l}1-2: 0.174 \\
1-3: 0.932 \\
2-3: 0.344\end{array}$ \\
\hline Post-cardiotomy & $20(5.9 \%)$ & $22(7.5 \%)$ & $7(14.3 \%)$ & $\begin{array}{l}1-2: 0.562 \\
1-3: 0.031 \\
2-3: 0.201\end{array}$ \\
\hline Others & $110(32.4 \%)$ & $12(23.7 \%)$ & $11(22.4 \%)$ & \\
\hline $\mathrm{OHCA}$ & $87(25.6 \%)$ & $15(16.1 \%)$ & $3(6.1 \%)$ & $\begin{array}{l}1-2: 0.057 \\
1-3: 0.003 \\
2-3: 0.090\end{array}$ \\
\hline Low-flow duration (min) & $40.3 \pm 21.2$ & $41.0 \pm 18.6$ & $44.3 \pm 26.6$ & $\begin{array}{l}1-2: 0.781 \\
1-3: 0.231 \\
2-3: 0.382\end{array}$ \\
\hline
\end{tabular}

CKD chronic kidney disease, NYHA New York Heart Association, COPD chronic obstructive pulmonary disease, CVA cerebral vascular attack, TIA transient ischemic attack, ACS acute coronary syndrome, CPR cardiopulmonary resuscitation, ECMO extracorporeal membrane oxygenation, OCHA out-of-hospital cardiac arrest, low-flow duration duration between CPR and ECMO run, CCI Charlson Comorbidity Index

suggest that a relatively high percentage of elderly patients with acute illness rather than chronic illness were referred for ECPR. The percentage of OHCA decreased as the age increased in the three groups (25.6\%, $16.1 \%$, and $6.1 \%$ in groups 1,2 , and 3 , respectively), which possibly reflected clinical discretion 
against ECPR use for the elderly patients who experienced OHCA. The LFDs of the three groups were comparable $(40.3 \pm 21.2,41.0 \pm 18.6$, and $44.3 \pm 26.6 \mathrm{~min}$ in groups 1,2 , and 3 , respectively).

The hospital outcomes for all the study patients are listed in Table 2. In brief, $44.2 \%$ of the patients were weaned off ECMO support, and $29.7 \%$ survived to hospital discharge. CPC 1, 2, 3, 4, and 5 account for $25.3 \%$, $0.8 \%, 1.0 \%, 2.5 \%$, and $70.3 \%$, respectively, with a total of $26.1 \%$ exhibiting favorable neurologic outcomes with CPC 1 or 2. Specifically, the ECMO weaning rate was higher in group 1 than in group $3(47.6 \%, 39.8 \%$, and $28.6 \%$ for groups 1,2 , and 3 , respectively; $P=0.012$ between groups 1 and 3 ). The hospital survival rate was higher in group 1 than in group $3(32.1 \%, 26.9 \%$, and $18.4 \%$ in groups 1,2 , and 3 , respectively; $P=0.052$ between groups 1 and 3). However, the rates of favorable neurologic outcomes (CPC 1, 2 at discharge) did not differ considerably among the three groups $(27.6 \%$, $24.7 \%$, and $18.4 \%$ for groups 1,2 , and 3, respectively; $P=0.575,0.169$, and 0.390 between groups 1 and 2,1 and 3 , and 2 and 3, respectively). The average ECMO support duration was lower in group 3 compared with group 1 and 2 (4.3, 3.0, 1, and 7 days for group 1, 2, and $3)$. After adjusting for their respective ECMO wean-off rate $(47.6 \%, 39.8 \%$, and $28.6 \%$ for group 1,2 , and 3$)$, the adjusted ECMO support duration was higher for the younger group (9.0, 7.5, and 5.9 days for group 1, 2, and
3), indicating longer ECMO support for young patients than old patients.

The overall 3-year survival rates were lower among the group 3 patients $(11.4 \%)$ than among the group 1 (25.5\%, $P<0.001)$ or group $2(24.7 \%, P=0.064)$ patients (Fig. 2 ). The 3-year survival probabilities of the patients who survive to hospital discharge were all $>60 \%$ in the three groups $(78.7 \%, 92.0 \%$, and $64.8 \%$ in groups 1,2 , and 3 , respectively).

Risk factor analysis for favorable neurologic outcomes with univariate logistic regression analysis revealed that CPR due to sepsis, LFDs, and early year of ECPR were potential risk factors for favorable neurologic outcomes (all with $P<0.15$ ) (Table 3). Notably, age was not a significant factor affecting favorable neurologic outcomes [odds ratio (OR) of 0.96 for every 10-year increment, $P=0.573$. The multivariate logistic regression analysis revealed that only CPR due to sepsis and prolonged LFDs exhibited negative effects on favorable neurologic outcomes $(P=0.013, P<0.001$, respectively), whereas early year of ECPR exhibited negative effects on favorable outcomes $(P=0.086)$.

Because age and LFD were two prominent clinical factors that could be collected immediately at the site of $\mathrm{CPR}$, the patients were divided into subgroups according to age and LFD to evaluate the effect of the interaction between age and LFD on favorable neurologic outcomes (Fig. 3). The favorable neurologic outcome rates were $34.6 \%, 24.1 \%$, and $13.2 \%$ among the patients with LFDs

Table 2 Outcome of ECPR

\begin{tabular}{|c|c|c|c|c|}
\hline & $18-65$ years old (340) & $65-75$ years old (93) & $>75$ years old $(49)$ & $P$ \\
\hline ECMO days & $4.3 \pm 6.8$ & $3.0 \pm 4.0$ & $1.7 \pm 1.8$ & $\begin{array}{l}1-2: 0.086 \\
1-3: 0.009 \\
2-3: 0.033\end{array}$ \\
\hline Ventilator days & $12.3 \pm 21.4$ & $7.6 \pm 11.5$ & $4.0 \pm 7.7$ & $\begin{array}{l}1-2: 0.042 \\
1-3: 0.007 \\
2-3: 0.049\end{array}$ \\
\hline ICU days & $14.2 \pm 20.9$ & $9.4 \pm 13.5$ & $6.6 \pm 12.6$ & $\begin{array}{l}1-2: 0.037 \\
1-3: 0.016 \\
2-3: 0.239\end{array}$ \\
\hline Hospitalization days & $24.3 \pm 36.3$ & $15.9 \pm 25.1$ & $10.3 \pm 22.1$ & $\begin{array}{l}1-2: 0.036 \\
1-3: 0.009 \\
2-3: 0.195\end{array}$ \\
\hline Weaned off ECMO & $162(47.6 \%)$ & $37(39.8 \%)$ & $14(28.6 \%)$ & $\begin{array}{l}1-2: 0.178 \\
1-3: 0.012 \\
2-3: 0.187\end{array}$ \\
\hline Hospital survival at discharge & 109 (32.1\%) & $25(26.9 \%)$ & $9(18.4 \%)$ & $\begin{array}{l}1-2: 0.339 \\
1-3: 0.052 \\
2-3: 0.260\end{array}$ \\
\hline $\begin{array}{l}\text { Favorable neurologic outcome (CPC } 1, \\
2 \text { at discharge) }\end{array}$ & $94(27.6 \%)$ & $23(24.7 \%)$ & $9(18.4 \%)$ & $\begin{array}{l}1-2: 0.575 \\
1-3: 0.169 \\
2-3: 0.390\end{array}$ \\
\hline
\end{tabular}

Data are presented by age group

CPC cerebral performance category 

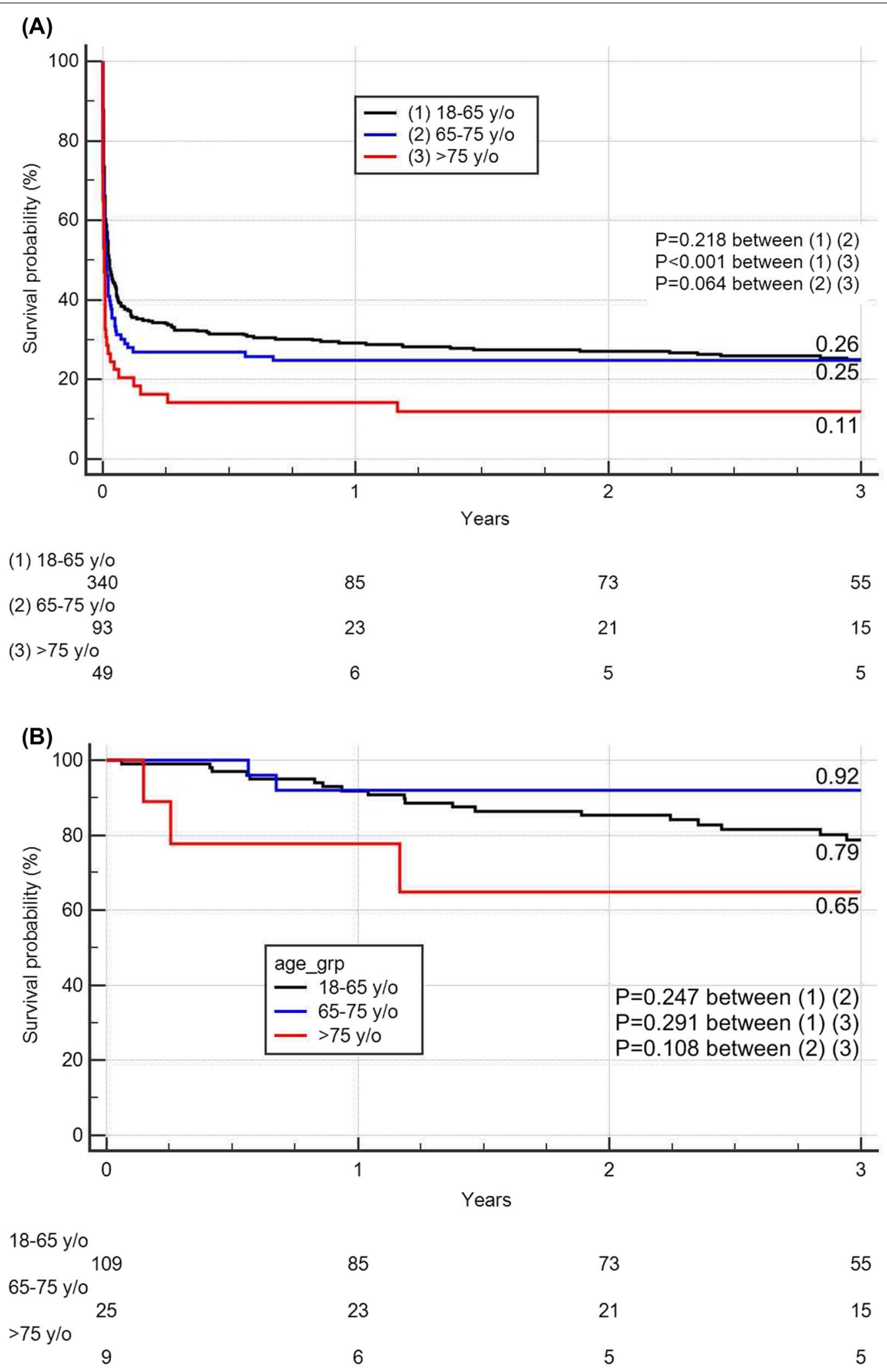

Fig. 2 Kaplan-Meier survival curve for extracorporeal cardiopulmonary resuscitation in different age groups for $\mathbf{a}$ all study patients and $\mathbf{b}$ hospital survivors 
Table 3 Logistic regression analysis for hospital discharge with favorable neurologic function $(C P C-1,2)$

\begin{tabular}{|c|c|c|c|c|}
\hline & \multicolumn{2}{|l|}{ Univariate analysis } & \multicolumn{2}{|l|}{ Multivariate analysis } \\
\hline & OR $(95 \% \mathrm{Cl})$ & $P$ & OR $(95 \% \mathrm{Cl})$ & $P$ \\
\hline Female gender & $0.95(0.59-1.54)$ & 0.845 & - & - \\
\hline $\mathrm{CCl}$ & $0.93(0.98-1.04)$ & 0.205 & - & - \\
\hline Age (+10 years) & $0.96(0.84-1.10)$ & 0.573 & - & - \\
\hline Age $>65$ years old & $0.76(0.48-1.21)$ & 0.245 & - & - \\
\hline Age $>75$ years old & $0.61(0.29-1.29)$ & 0.195 & - & - \\
\hline CPR due to ACS & $1.21(0.80-1.83)$ & 0.359 & - & - \\
\hline CPR due to sepsis & $0.23(0.07-0.77)$ & $0.017^{*}$ & $0.21(0.06-0.73)$ & 0.013 \\
\hline CPR due to post-cardiotomy & $1.38(0.65-2.93)$ & 0.394 & - & - \\
\hline Low-flow duration (+ 10 min) & $0.80(0.71-0.90)$ & $<0.001^{*}$ & $0.79(0.70-0.89)$ & $<0.001$ \\
\hline OHCA & $0.85(0.51-1.41)$ & 0.539 & - & - \\
\hline \multicolumn{5}{|l|}{ ECPR period } \\
\hline Period 1 (2006-2009) & $0.69(0.43-1.10)$ & $0.119^{*}$ & $0.65(0.39-1.06)$ & 0.086 \\
\hline Period 2 (2010-2013) & $1.39(0.91-2.11)$ & 0.130 & - & - \\
\hline Period 3 (2014-2016) & $1.00(0.66-1.51)$ & 0.983 & - & - \\
\hline
\end{tabular}

Variables with $P<0.15$ by univariate analysis (marked with an asterisk) were subjected to multivariate analysis. The multivariate logistic regression analysis was set with entry and removal $P$ values of 0.10 and 0.15 , respectively

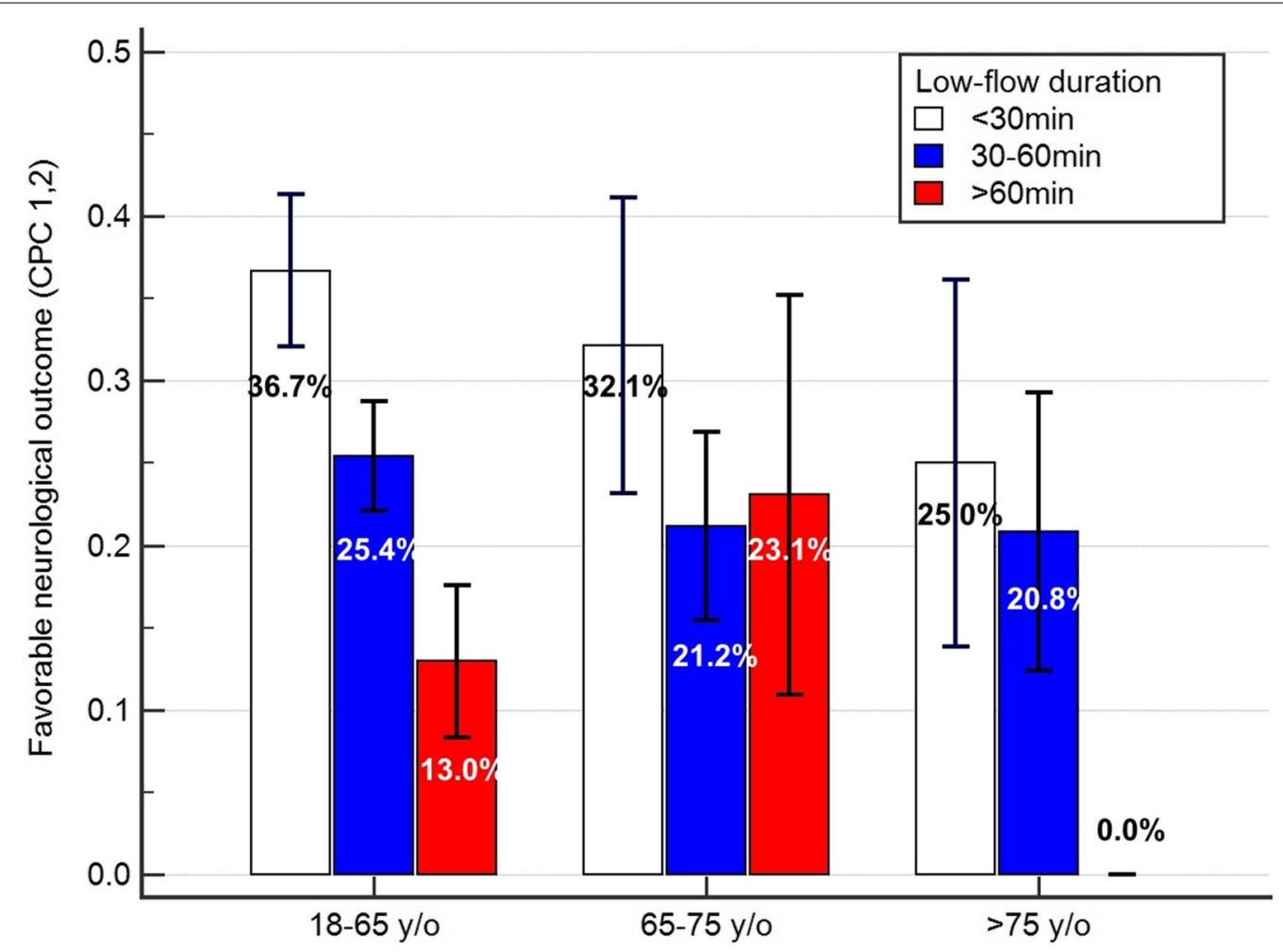

Fig. 3 Effects of interplay between age and low-flow duration on favorable neurologic outcome rates after extracorporeal cardiopulmonary resuscitation

of $<30,30-60$, and $>60 \mathrm{~min}$, respectively $(P=0.002$.) As shown in Fig. 3, group 1 had a mean LFD of $<30 \mathrm{~min}$ and was associated with the highest neurologic outcome rate
(36.7\%), whereas group 3 had a mean LFD of $>60 \mathrm{~min}$ and was associated with the lowest neurologic outcome rate $(0.0 \%)$. Notably, the rate of favorable neurologic 
outcomes in group 3 with a mean LFD of $<30 \mathrm{~min}$ (25.0\%) was similar to the rate in group 1 with a mean LFD of $>60 \mathrm{~min}(13.0 \%)(P=0.249)$, thus indicating that under the current cardiopulmonary resuscitation protocol and ECPR selection criteria, patients aged $>75$ years still have a $25 \%$ chance of surviving the indexed cardiac arrest episode.

To elucidate whether the patients who experienced IHCA and OHCA had different neurologic outcomes depending on their ages or LFDs, the results of subgroup analysis are shown in Supplement Fig. 2. The results revealed that under the current selection criteria for ECMO use (Supplement Table 1), the rate of favorable neurologic outcomes in the patients who experienced OHCA was almost equal to that in the patients who experienced IHCA in each subgroup of age and LFD.

\section{Discussion}

Age and LFD are the two most determinative factors in the emergency scenario of CPR. Studies have shown that both advanced age and prolonged LFDs are associated with poor neurologic outcomes [3, 4, 13]; therefore, both are generally considered contraindications for ECMO use in CPR [24]. However, whether relatively young patients with long LFDs or elderly patients with relatively short LFDs have acceptable chances of survival after ECPR has not been studied. This article reports real-world data regarding the effects of interplay between age and LFD on favorable neurologic outcome rates after ECPR. The results revealed that for LFDs of $<60 \mathrm{~min}$, ECPR was associated with an acceptable hospital outcome regardless of age, whereas for LFDs of $>60 \mathrm{~min}$, ECPR was associated with poor neurologic outcomes only for the patients aged $>75$ years. These findings provide objective references for shared decisions regarding the use of ECPR in elderly patients or patients with prolonged cardiac massage durations.

The results of the present study revealed that for elderly patients with LFDs of $<60 \mathrm{~min}$, the neurologic outcomes of ECPR were not as poor as previously thought; this finding was inconsistent with the Extracorporeal Life Support Organization (ELSO) guidelines, which define advanced age as an absolute or relative contraindication for ECPR [24]. Previous studies have indicated that intrinsically old age is not a risk factor for hospital mortality after ECPR of either IHCA [25] or OHCA [9, $26]$. By contrast, the underlying cause of cardiac collapse affects hospital survival more than age does. As shown in Table 1, the proportion of patients with ACS as the etiology of cardiac arrest was higher in the elderly ECPR patients than in the younger patients, whereas the proportion of patients with chronic heart failure as the etiology of cardiac arrest was higher in the younger patients than in the elderly ECPR patients. Because of the rapid door-to-balloon protocol currently practiced, ECMO use for ACS has been reported to be associated with acceptable outcomes [27-29], if treated promptly. By contrast, ECPR due to septic shock, which is usually associated with a complex underlying comorbidity, was associated with a relatively low rate of favorable outcomes, as reported in the present study and in a previous study [30]. The proportion of patients with sepsis as the etiology of cardiac arrest in this study was lower in the elderly patients (4.3\% and $8.2 \%$ in groups 2 and 3, respectively) than in the younger patients ( $8.5 \%$ in group 1$)$.

Traditional CPR for patients who experienced OHCA and IHCA was associated with low hospital survival rates, namely $7.2-9.9 \%[10,31,32]$ and $14.2-15.7 \%$ [33, 34], respectively. Notably, most of the patients achieving return of spontaneous circulation (ROSC) were resuscitated for $<30 \mathrm{~min}(42.5 \%)$, whereas only $6 \%$ of patients achieving ROSC were resuscitated for $>30 \mathrm{~min}$ [33].

The ECPR program was specifically designed for patients who receive sustained CPR for $>30 \mathrm{~min}$ and for improving their chances of hospital survival $[3,18,20]$. Hospital survival rate has been found to improve slightly from 29.0 to $29.7 \%$ after ECPR $[1,20]$. In addition, studies have compared the efficacy of ECPR with conventional $\mathrm{CPR}$ in preventing long-term neurologic damage and have shown that ECPR provides markedly better neurologic protection than does traditional CPR [35]. However, ECMO per se is also associated with the risks of neurologic injury [36, 37], including anoxic brain injury, intracranial hemorrhage, and ischemic stroke. Old age, prior $\mathrm{CPR}$, and low arterial pressure during ECMO support are associated with poor neurologic outcomes [36] and hospital mortality [13].

Animal studies have also demonstrated that responses to neurologic injuries after ischemia are different in young and old animals [6]; young and old animals exhibit relatively high levels of hypoxic and apoptotic injury, respectively. A previous study [38] on the pathologic examination of patients with cardiac arrest revealed that the severity of hypoxic ischemic brain injury is correlated with prolonged survival time after cardiac arrest instead of age, hypertension, or diabetes. These findings indicate that after cardiac arrest, hypoxic brain injury is a complicated and reactive process, which involves an interaction among initial hypoxic damage, reperfusion injury, subsequent oxidative reactions, and apoptotic reactions $[39,40]$. New strategies to prevent or alleviate neurologic injury after ECPR, including pharmacologic vasodilation [41], post-ECPR conditioning [39, 42], or systemic $[43,44]$ or intracranial therapeutic hypothermia [45], are potential innovative treatments to improve neurologic outcomes after ECPR. 
Elderly patients aged > 65 years accounted for $29.4 \%$ of all ECPR cases in the present study, which was similar to ELSO registry data [11], but the percentage was considerably lower than the traditional CPR registry data of $60-70 \%[10,32,34]$. This indicates that clinical discretion during CPR consultation favors young patients for ECMO support. In addition to age, patients with low CCI scores (J Clin Med 7(10):313; https://doi.org/10.3390/ jcm7100313 (2018) (registering DOI) ) who had received short-duration cardiac massages, high-quality CPR from EMTs [31], or ACS in etiology (according to initial assessments) had relatively high chances of receiving ECMO during CPR as a temporary cardiopulmonary support for the definite diagnosis and treatment and later recovery of cardiopulmonary and neurologic functions.

As shown in Fig. 2, the 3-year survival probabilities were $79 \%, 92 \%$, and $65 \%$ in groups 1,2 , and 3 , respectively. Late mortality in CPR patients after hospital discharge has emerged as a new problem. Get With The Guidelines-Resuscitation registry data revealed that only $60 \%$ of IHCA survivors survived at 1 year [46]. Continuous morbidity and mortality may be attributed to a relatively high frequency of posttraumatic stress disorder, low levels of well-being [47] and cognitive function, and impaired activities of daily living [48] in OHCA survivors. These findings indicate that a post-ECPR specialized joint care program is necessary for ECPR survivors.

\section{Limitations}

First, the number of study patients was low, which may have affected the data analysis. Second, the present study was not a prospective randomized study and performed in a single institute, and there may have been selection bias in both the young and old patients for ECMO use that favored patients who had fewer preexisting comorbidities, shorter period of cardiac collapse time, or received better quality of CPR. Finally, the outcome of ECPR was determined by a combination of ECMO and pre-ECMO conditions, including extensiveness and endurance of CPR. Khan et al. [49] reported that the duration of CPR for non-ROSC patients was lower in patients aged $>65$ years and with pre-existing comorbidities.

\section{Clinical implications}

Considering hospital survival and neurologic outcome rates, ECPR is a suitable treatment for either elderly patients with short cardiac arrest durations or young patients with long cardiac arrest durations. However, ECPR is not recommended for use in elderly patients with prolonged cardiac arrest duration owing to the relatively poor associated outcomes.
Electronic supplementary material

The online version of this article (https://doi.org/10.1007/s00134-018-5496-y) contains supplementary material, which is available to authorized users.

\section{Acknowledgements}

The study was partially supported by the research fund of the Ministry of Science and Technology, Taiwan (MOST 106-2314-B-002-154-MY3 and MOST 106-2314-B-002-234-MY3).

\section{Compliance with ethical standards}

\section{Conflicts of interest}

All the authors declare that they have no conflicts of interest.

\section{Open Access}

This article is distributed under the terms of the Creative Commons Attribution-NonCommercial 4.0 International License (http://creativecommons.org/ licenses/by-nc/4.0/), which permits any noncommercial use, distribution, and reproduction in any medium, provided you give appropriate credit to the original author(s) and the source, provide a link to the Creative Commons license, and indicate if changes were made.

Received: 16 August 2018 Accepted: 3 December 2018

Published online: 13 December 2018

\section{References}

1. Thiagarajan RR, Barbaro RP, Rycus PT et al (2017) Extracorporeal Life Support Organization Registry International Report 2016. ASAIO J 63:60-67. https://doi.org/10.1097/MAT.0000000000000475

2. Mentzelopoulos SD, Slowther A-M, Fritz Z et al (2018) Ethical challenges in resuscitation. Intens Care Med 44:703-716. https://doi.org/10.1007/ s00134-018-5202-0

3. Chen Y-S, Lin J-W, Yu H-Y et al (2008) Cardiopulmonary resuscitation with assisted extracorporeal life-support versus conventional cardiopulmonary resuscitation in adults with in-hospital cardiac arrest: an observational study and propensity analysis. Lancet 372:554-561. https://doi. org/10.1016/S0140-6736(08)60958-7

4. Wengenmayer T, Rombach S, Ramshorn F et al (2017) Influence of lowflow time on survival after extracorporeal cardiopulmonary resuscitation (eCPR). Crit Care 21:157. https://doi.org/10.1186/s13054-017-1744-8

5. Llorente IL, Burgin TC, Pérez-Rodríguez D et al (2013) Unfolded protein response to global ischemia following $48 \mathrm{~h}$ of reperfusion in the rat brain: the effect of age and meloxicam. J Neurochem 127:701-710. https://doi. org/10.1111/jnc.12337

6. Anuncibay-Soto B, Pérez-Rodríguez D, Llorente IL et al (2014) Agedependent modifications in vascular adhesion molecules and apoptosis after 48-h reperfusion in a rat global cerebral ischemia model. Age (Dordr) 36:9703. https://doi.org/10.1007/s11357-014-9703-7

7. Mendiratta P, Wei JY, Gomez A et al (2013) Cardiopulmonary resuscitation requiring extracorporeal membrane oxygenation in the elderly: a review of the extracorporeal life support organization registry. ASAIO J 59:211-215. https://doi.org/10.1097/MAT.0b013e31828fd6e5

8. Ouweneel DM, Schotborgh JV, Limpens J et al (2016) Extracorporeal life support during cardiac arrest and cardiogenic shock: a systematic review and meta-analysis. Intens Care Med 42:1922-1934. https://doi. org/10.1007/s00134-016-4536-8

9. Debaty G, Babaz V, Durand M et al (2017) Prognostic factors for extracorporeal cardiopulmonary resuscitation recipients following out-of-hospital refractory cardiac arrest. A systematic review and meta-analysis. Resuscitation 112:1-10. https://doi.org/10.1016/j.resuscitation.2016.12.011

10. Kitamura T, Iwami T, Atsumi T et al (2018) The profile of Japanese Association for Acute Medicine-out-of-hospital cardiac arrest registry in 2014-2015. Acute Med Surg 5:249-258. https://doi.org/10.1002/ ams 2.340

11. Haas NL, Coute RA, Hsu CH et al (2017) Descriptive analysis of extracorporeal cardiopulmonary resuscitation following out-of-hospital cardiac 
arrest-An ELSO registry study. Resuscitation 119:56-62. https://doi. org/10.1016/j.resuscitation.2017.08.003

12. Ebell MH, Jang W, Shen Y et al (2013) Development and validation of the Good Outcome Following Attempted Resuscitation (GO-FAR) score to predict neurologically intact survival after in-hospital cardiopulmonary resuscitation. JAMA Intern Med 173:1872-1878. https://doi.org/10.1001/ jamainternmed.2013.10037

13. Schmidt M, Burrell $A$, Roberts L et al (2015) Predicting survival after ECMO for refractory cardiogenic shock: the survival after veno-arterial-ECMO (SAVE)-score. Eur Heart J 36:2246-2256. https://doi.org/10.1093/eurhe artj/ehv194

14. Chen Y-S, Ko W-J, Chi N-H et al (2004) Risk factor screening scale to optimize treatment for potential heart transplant candidates under extracorporeal membrane oxygenation. Am J Transpl 4:1818-1825. https ://doi.org/10.1111/j.1600-6143.2004.00578.x

15. Chen Y-S, Yu H-Y, Huang S-C et al (2005) Experience and result of extracorporeal membrane oxygenation in treating fulminant myocarditis with shock: what mechanical support should be considered first? J Heart Lung Transpl 24:81-87. https://doi.org/10.1016/j.healun.2003.09.038

16. Chen Y-S, Yu H-Y, Huang S-C et al (2008) Extracorporeal membrane oxygenation support can extend the duration of cardiopulmonary resuscitation. Crit Care Med 36:2529-2535. https://doi.org/10.1097/CCM.0b013 e318183f491

17. Lin J-W, Wang M-J, Yu H-Y et al (2010) Comparing the survival between extracorporeal rescue and conventional resuscitation in adult in-hospital cardiac arrests: propensity analysis of three-year data. Resuscitation 81:796-803. https://doi.org/10.1016/j.resuscitation.2010.03.002

18. Chen YS, Chao A, Yu HY et al (2003) Analysis and results of prolonged resuscitation in cardiac arrest patients rescued by extracorporeal membrane oxygenation. JAC 41:197-203. https://doi.org/10.1016/S0735 -1097(02)02716-X

19. Huang S-C, Wu E-T, Wang C-C et al (2012) Eleven years of experience with extracorporeal cardiopulmonary resuscitation for pediatric patients with in-hospital cardiac arrest. Resuscitation 83:710-714. https://doi. org/10.1016/j.resuscitation.2012.01.031

20. Wang C-H, Chou N-K, Becker LB et al (2014) Improved outcome of extracorporeal cardiopulmonary resuscitation for out-of-hospital cardiac arrest-a comparison with that for extracorporeal rescue for in-hospital cardiac arrest. Resuscitation 85:1219-1224. https://doi.org/10.1016/j.resus citation.2014.06.022

21. Lerner EB, Rea TD, Bobrow BJ et al (2012) Emergency medical service dispatch cardiopulmonary resuscitation prearrival instructions to improve survival from out-of-hospital cardiac arrest: a scientific statement from the American Heart Association. Circulation 125:648-655. https://doi. org/10.1161/CIR.0b013e31823ee5fc

22. Charlson ME, Charlson RE, Peterson JC et al (2008) The Charlson comorbidity index is adapted to predict costs of chronic disease in primary care patients. J Clin Epidemiol 61:1234-1240. https://doi.org/10.1016/j.jclinepi.2008.01.006

23. Quan H, Li B, Couris CM et al (2011) Updating and validating the Charlson comorbidity index and score for risk adjustment in hospital discharge abstracts using data from 6 countries. Am J Epidemiol 173:676-682. https ://doi.org/10.1093/aje/kwq433

24. (2014) Life Extracorporeal Support Organization (ELSO) Guidelines for ECPR Cases. 1-4.

25. D'Arrigo S, Cacciola S, Dennis M et al (2017) Predictors of favourable outcome after in-hospital cardiac arrest treated with extracorporeal cardiopulmonary resuscitation: a systematic review and meta-analysis. Resuscitation 121:62-70. https://doi.org/10.1016/j.resuscitation.2017.10.005

26. Ryu J-A, Cho YH, Sung K et al (2015) Predictors of neurological outcomes after successful extracorporeal cardiopulmonary resuscitation. BMC Anesthesiol 15:26. https://doi.org/10.1186/s12871-015-0002-3

27. Chung S-Y, Tong M-S, Sheu J-J et al (2016) Short-term and long-term prognostic outcomes of patients with ST-segment elevation myocardial infarction complicated by profound cardiogenic shock undergoing early extracorporeal membrane oxygenator-assisted primary percutaneous coronary intervention. Int J Cardiol 223:412-417. https://doi. org/10.1016/j.ijcard.2016.08.068

28. Esposito M, Bader Y, Pedicini R et al (2017) The role of acute circulatory support in ST-segment elevation myocardial infarction complicated by cardiogenic shock. Indian Heart J 69:668-674. https://doi.org/10.1016/j. ihj.2017.05.011
29. Sung P-H, Wu C-J, Yip H-K (2016) Is extracorporeal membrane oxygenator a new weapon to improve prognosis in patients with profound cardiogenic shock undergoing primary percutaneous coronary intervention? Circ J 80:572-578. https://doi.org/10.1253/circj.CJ-15-1398

30. Aubin H, Petrov G, Dalyanoglu H et al (2016) A suprainstitutional network for remote extracorporeal life support: a retrospective cohort study. JACC Heart Fail 4:698-708. https://doi.org/10.1016/j.jchf.2016.03.018

31. Sun J-T, Chiang W-C, Hsieh M-J et al (2018) The effect of the number and level of emergency medical technicians on patient outcomes following out of hospital cardiac arrest in Taipei. Resuscitation 122:48-53. https:// doi.org/10.1016/j.resuscitation.2017.11.048

32. Nehme Z, Bernard S, Cameron P et al (2015) Using a cardiac arrest registry to measure the quality of emergency medical service care: decade of findings from the Victorian Ambulance Cardiac Arrest Registry. Circ Cardiovasc Qual Outcomes 8:56-66. https://doi.org/10.1161/CIRCOUTCOMES.114.001185

33. Goldberger ZD, Chan PS, Berg RA et al (2012) Duration of resuscitation efforts and survival after in-hospital cardiac arrest: an observational study. Lancet 380:1473-1481. https://doi.org/10.1016/S0140-6736(12)60862-9

34. Wang C-H, Huang C-H, Chang W-T et al (2018) associations between body size and outcomes of adult in-hospital cardiac arrest: a retrospective Cohort study. Resuscitation 130:67-72. https://doi.org/10.1016/j. resuscitation.2018.07.006

35. Shin TG, Jo IJ, Sim MS et al (2013) Two-year survival and neurological outcome of in-hospital cardiac arrest patients rescued by extracorporeal cardiopulmonary resuscitation. Int J Cardiol 168:3424-3430. https://doi. org/10.1016/j.jjcard.2013.04.183

36. Mateen FJ, Muralidharan R, Shinohara RT et al (2011) Neurological injury in adults treated with extracorporeal membrane oxygenation. Arch Neurol 68:1543-1549. https://doi.org/10.1001/archneurol.2011.209

37. Lorusso R, Barili F, Mauro MD et al (2016) In-hospital neurologic complications in adult patients undergoing venoarterial extracorporeal membrane oxygenation: results from the extracorporeal life support organization registry. Crit Care Med 44:e964-e972. https://doi.org/10.1097/CCM.00000 00000001865

38. Md AH, Md HG, Md JDY, Md SO (2014) Hypoxic ischemic brain injury following in hospital cardiac arrest-lessons from autopsy. J Forensic Leg Med 23:84-86. https://doi.org/10.1016/j.jflm.2014.02.003

39. Adstamongkonkul D, Hess DC (2017) Ischemic conditioning and neonatal hypoxic ischemic encephalopathy: a literature review. Cond Med 1:9-16

40. Menyhárt Á, Zölei-Szénási D, Puskás T et al (2017) Age or ischemia uncouples the blood flow response, tissue acidosis, and direct current potential signature of spreading depolarization in the rat brain. Am J Physiol Heart Circ Physiol 313:H328-H337. https://doi.org/10.1152/ajpheart.00222.2017

41. Perkins GD, Ji C, Deakin CD et al (2018) A randomized trial of epinephrine in out-of-hospital cardiac arrest. N Engl J Med. https://doi.org/10.1056/ NEJMoa1806842

42. Bonova P, Burda J, Danielisova V et al (2013) Delayed post-conditioning reduces post-ischemic glutamate level and improves protein synthesis in brain. Neurochem Int 62:854-860. https://doi.org/10.1016/j.neuin t.2013.02.019

43. Meybohm P, Gruenewald M, Albrecht M et al (2009) Hypothermia and postconditioning after cardiopulmonary resuscitation reduce cardiac dysfunction by modulating inflammation, apoptosis and remodeling PLoS ONE 4:e7588-e7610. https://doi.org/10.1371/journal.pone.0007588

44. BenedekT, Popovici MM, Glogar D (2016) Extracorporeal life support and new therapeutic strategies for cardiac arrest caused by acute myocardial infarction - a critical approach for a critical condition. J Crit Care Med (Targu Mures) 2:164-174. https://doi.org/10.1515/jccm-2016-0025

45. Wang C-H, Lin Y-T, Chou H-W et al (2017) Novel approach for independent control of brain hypothermia and systemic normothermia: cerebral selective deep hypothermia for refractory cardiac arrest. J Neurointerv Surg 9:e32. https://doi.org/10.1136/neurintsurg-2016-012806.rep

46. Thompson LE, Chan PS, Tang F et al (2018) Long-term survival trends of medicare patients after in-hospital cardiac arrest: insights from get with the guidelines-resuscitation ${ }^{\circledR}$. Resuscitation 123:58-64. https://doi. org/10.1016/j.resuscitation.2017.10.023

47. Viktorisson A, Sunnerhagen KS, Pöder U et al (2018) Well-being among survivors of out-of-hospital cardiac arrest: a cross-sectional retrospective study in Sweden. BMJ Open 8:e021729. https://doi.org/10.1136/bmjop en-2018-021729 
48. Lundgren-Nilsson Å, Rosén H, Hofgren C, Sunnerhagen KS (2005) The first year after successful cardiac resuscitation: function, activity, participation and quality of life. Resuscitation 66:285-289. https://doi.org/10.1016/j. resuscitation.2005.04.001
49. Khan AM, Kirkpatrick JN, Yang L et al (2014) Age, sex, and hospital factors are associated with the duration of cardiopulmonary resuscitation in hospitalized patients who do not experience sustained return of spontaneous circulation. J Am Heart Assoc 3:e001044. https://doi.org/10.1161/JAHA.114.001044 